\title{
新型侧链含氟苯并噁嗪的合成及其性能的研究
}

\author{
吴建平 ${ }^{a, b}$ 赖 华 $*, b$ 才 屾 ${ }^{b}$ 金凯凯 ${ }^{b}$ \\ 袁 超 ${ }^{b}$ 房 强*,b \\ ( ${ }^{a}$ 华东理工大学材料科学与工程学院 上海 200237) \\ ${ }^{b}$ 中国科学院上海有机化学研究所 上海 200032)
}

\begin{abstract}
摘要 采用简洁的方法, 合成了一种新型侧链含长氟碳链的苯并噁嗪单体(M1), 并研究了其热固化反应和固化产物的 性能. 结果表明, M1 的起始固化温度为 $257{ }^{\circ} \mathrm{C}$, 但当加入 $5 \mathrm{wt} \%$ 的咪唑催化剂后, 其固化起始温度降低至 $183{ }^{\circ} \mathrm{C}$. M1 聚合物具有优异的热稳定性，在氮气氛中其 $5 \%$ 和 $10 \%$ 热重损失温度分别为 337 和 $382{ }^{\circ} \mathrm{C}, 800{ }^{\circ} \mathrm{C}$ 碳残余率为 $45.9 \%$. 该固化树脂亦具有良好的介电性能, 在 $1 \mathrm{MHz}$ 频率下其介电常数为 3.78. 常温下固化树脂的吸水率小于 $1 \%$, 表面自由 能为 $6.98 \mathrm{mN} / \mathrm{m}$. 以上结果表明侧链含氟的苯并噁嗪有望成为一种性能优异的新型绝缘材料.
\end{abstract}

关键词 苯并噁嗪; 氟化物; 热稳定性; 介电常数; 吸水率; 表面性能

\section{Synthesis and Properties of the Polymer Based on Benzoxazine with Two Perfluorohexyl Side Chains}

\author{
Wu, Jianping ${ }^{a} \quad$ Lai, Hua $^{*, b}$ \\ Diao, Shen ${ }^{b}$ \\ Jin, Kaikai ${ }^{b}$ \\ Yuan, Chao ${ }^{b}$ \\ Fang, Qiang ${ }^{*, b}$ \\ ( ${ }^{a}$ East China University of Science \& Technology, School of Materials Science and Engineering, Shanghai 200237) \\ $\left({ }^{b}\right.$ Key Laboratory of Organofluorine Chemistry and Laboratory for Polymer Materials, Shanghai Institute of \\ Organic Chemistry, Chinese Academy of Sciences, Shanghai 200032)
}

\begin{abstract}
A new benzoxazine with two perfluorohexyl side chains (M1) was synthesized and its homopolymerization was investigated. The results showed that the curing of $\mathbf{M 1}$ was occurred at near $257{ }^{\circ} \mathrm{C}$, whereas the curing onset temperature can be lowered to $183{ }^{\circ} \mathrm{C}$ when a catalyst of $5 \mathrm{wt} \%$ imidazole was added. The cured M1 exhibited higher thermal stability with $5 \%$ and $10 \%$ weight loss temperatures of 337 and $382{ }^{\circ} \mathrm{C}$, and a char yield of $45.9 \%$, respectively. The cured M1 exhibited also good electrical properties with dielectric constant of 3.78 in a frequency of $1 \mathrm{MHz}$. The water absorption of the cured M1 was below $1 \%$, and the surface free energy was $6.98 \mathrm{mN} / \mathrm{m}$, respectively.
\end{abstract}

Keywords benzoxaizne; thermal stability; dielectric constant; water absorbance; surface property

苯并噁嗪作为一种新型的热固性树脂，具有固化时 无小分子释放、制品的固化收缩率小、孔隙率低、耐热 性和阻燃性高、机械加工性能好等优点, 受到产业界和 学术界的青睐, 部分品种已经应用于航空、航天和电子 电气等领域 ${ }^{[1 \sim 3]}$. 随着电子工业的发展, 对高耐热和低 介电常数材料的需求逐年增加 ${ }^{[4 \sim 6]}$, 而传统的苯并噁嗪 聚合物难以满足要求. 基于含氟聚合物具有优异的耐热 性能和介电性能, 因此将含氟单元引入苯并噁嗪, 可以 提高聚合物的热稳定性和介电性能 ${ }^{[7 \sim 9]}$. 然而, 大量的
研究工作主要集中在将含氟单元引入苯并噁溙的主 链 ${ }^{[10 ~ 12]}$, 而对侧链含氟苯并噁嗪研究很少. 特别是, 在 苯并噁嗪主链上引入全氟碳链时, 需要价格昂贵的含氟 底物如双碘代全氟烷烃等, 会大幅增加聚合物的合成成 本. 同时, 传统的一步溶液法合成含氟苯并噁嗪产率较 低，提纯过程复杂，产物纯度不高，生成较多的二聚体 和三聚体 ${ }^{[13]}$ 不利于对单体特性的研究.

我们最近的研究发现, 侧链含有氟碳链的改性不饱 和聚酯树脂, 具有较低的介电常数和高的热稳定性 ${ }^{[14]}$.

* E-mail: qiangfang@mail.sioc.ac.cn; laihua@mail.sioc.ac.cn

Received December 11, 2012; revised January 6, 2013; published online January 9, 2012.

Project supported by the Ministry of Science and Technology of China (No. 2011ZX02703).

国家科技部重大专项资金(No. 2011ZX02703)资助项目 
鉴于侧基含长氟碳链苯并噁嗪的研究较少, 我们利用三 步法 ${ }^{[15]}$ 合成了一种新型侧链含氟苯并啞嗪 M1, 该合成 方法具有较高的产率, 所得产物具有较高的纯度. 同时 研究了其固化行为和聚合物的物理性质, 研究发现, 相 比于类似结构的不含氟聚苯并噁嗪 ${ }^{[12], M 1}$ 聚合物具有 优异的耐热性能、介电性能、耐水性和表面性能. 本文 报道详细结果.

\section{1 结果与讨论}

\section{1 单体 M1 的合成与表征}

我们曾尝试采用传统的一步法合成含氟苯并噁嗪. 将含氟苯胺 2、甲醛溶液, 双酚 $\mathrm{A}$ 和溶剂甲苯加入三口 瓶中, 升温至 $100{ }^{\circ} \mathrm{C}$ 反应. 由于含氟苯胺 2 为弱胺, 反 应活性低, 通过薄层色谱(TLC) 只观察到少量的目标产 物, 并伴随大量副产物生成, 不利于的产物提纯. 同时, 反应介质的 $\mathrm{pH}$ 值对反应产率和速率也有很大的影响, 需要进一步的调控. 这些都增加了合成含氟苯并噁嗪的 难度. 因此, 采用三步法, 合成路径如 Scheme 1 所示. 即首先将 5,5'-亚甲基水杨醛 ${ }^{[16]}$ (1) 和 4-全氟己烷苯 胺 ${ }^{[17]}(2)$ 溶解在乙醇溶液中, 升温反应 $2 \mathrm{~h}$, 分离提纯, 以 $77.8 \%$ 的产率得到席夫碱 $\mathbf{3}$. 其次, 将席夫碱 $\mathbf{3}$ 混合在 乙醇溶液中, 用硼氢化钠将其还原生成化合物 4, 分离 提纯后产率达到 $99 \%$. 最后, 在化合物 4 的氯仿溶液中, 加入甲醛溶液进行关环反应，以 $98 \%$ 的产率成功得到目 标产物 M1.

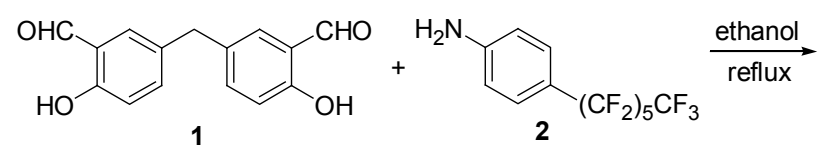<smiles>[R17]c1ccc(/N=C/c2cc(Cc3ccc(O)c(/C=N/c4ccc([R1])cc4)c3)ccc2O)cc1</smiles><smiles>[R]c1ccc(NCc2cc(Cc3ccc(O)c(CNc4ccc([R])cc4)c3)ccc2O)cc1</smiles><smiles>[R]c1ccc(N2COc3ccc(Cc4ccc5c(c4)CN(c4ccc([R])cc4)CO5)cc3C2)cc1</smiles>

Scheme 1

通过 ${ }^{1} \mathrm{H} \mathrm{NMR},{ }^{13} \mathrm{C} \mathrm{NMR},{ }^{19} \mathrm{~F}$ NMR 和元素分析等, 验证了单体 M1 化学结构. 图 1 为含氟苯并噁嗪单体 M1 的核磁氢谱和氟谱, 化学位移在 $\delta 4.64\left(\mathrm{ArCH}_{2} \mathrm{~N}\right)$ 和
$5.36\left(\mathrm{NCH}_{2} \mathrm{O}\right)$ 为噁嗪环的特征共振峰, $\delta 3.79$ 共振峰归 属于苯环之间亚甲基. $\delta 6.6 \sim 7.6$ 之间的共振峰归属于 苯环上氢, 核磁氟谱的六个不同化学位移上的共振峰也 对应于 $\left(\mathrm{CF}_{2}\right)_{5} \mathrm{CF}_{3}$ 不同碳上的氟原子. 同时, 元素分析结 果也与计算结果相吻合. 由以上分析, 验证了 Scheme 1 中苯并噁嗪 M1 单体化学结构.
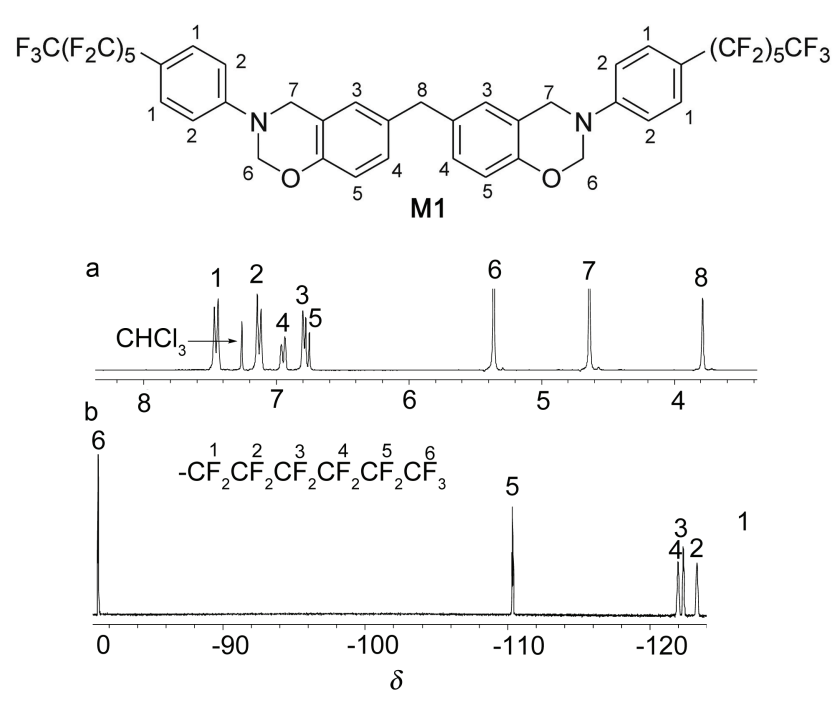

图 1 M1 单体的核磁氢谱(a)和氟谱(b)

Figure $1{ }^{1} \mathrm{H}$ NMR (a) and ${ }^{19} \mathrm{~F}$ NMR (b) spectra of M1 monomer

\section{$1.2 M 1$ 热固化行为的研究}

通过示差扫描量热法(DSC)研究 M1 的固化行为, 如图 2 所示. 其中在 $133{ }^{\circ} \mathrm{C}$ 为 $\mathbf{M 1}$ 的熔融吸热峰. M1 在 无固化剂条件下, 在 $257{ }^{\circ} \mathrm{C}$ 开始固化, 并在 $281{ }^{\circ} \mathrm{C}$ 出现 最大放热峰, 固化放热焓为 $90 \mathrm{~J} / \mathrm{g}$. 加入 $5 \mathrm{wt} \%$ 的固化剂 咪唑后, 其固化起始温度降低至 $183{ }^{\circ} \mathrm{C}$, 并在 $212{ }^{\circ} \mathrm{C}$ 出 现最大放热峰. 同时, 固化放热峰变宽, 使得 M1 的加 工窗口变宽, 加工性能得到改善. 可以观察到两个放热

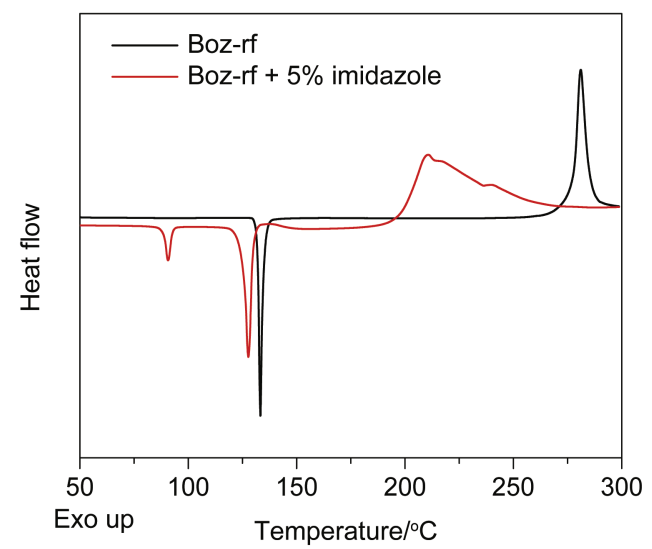

图 2 单体 M1 及加入 $5 \mathrm{wt} \%$ 咪唑后的 DSC 固化曲线 Figure 2 DSC traces of M1 monomer and M1 with 5 wt $\%$ imidazole respectively 
峰: 第一个峰在 $180{ }^{\circ} \mathrm{C}$ 至 $230{ }^{\circ} \mathrm{C}$ 之间, 为咪唑催化苯并 噁嗪单体在较低的温度下开环聚合; 另一个峰高于 230 ${ }^{\circ} \mathrm{C}$, 为热催化苯并噁嗪开环和生成的酚羟基继续催化 M1 单体开环聚合双重作用的结果 ${ }^{[18]}$.

M1 在不同温度下固化的 FT-IR 图谱如图 3 所示, 噁嗪环的特征吸收峰 $(\mathrm{N}-\mathrm{C}-\mathrm{O}$ 伸缩振动)在 960 930 $\mathrm{cm}^{-1}$ 之间, 并随着固化温度的不断升高而逐渐减弱, 说 明噁嗪环开环聚合. 当温度上升至 $220{ }^{\circ} \mathrm{C}$ 并保持 $2 \mathrm{~h}$ 后, 吸收峰几乎消失, 说明固化反应完全. $1500 \mathrm{~cm}^{-1}$ 为 1,2,4-苯环三取代特征吸收峰, 并随着固化温度升高而 逐渐消失. 随着固化反应的进行, $1660 \mathrm{~cm}^{-1}$ 出现 $1,2,3-$ 苯环三取代特征吸收峰. 1038 和 $1228 \mathrm{~cm}^{-1}(\mathrm{Ar}-\mathrm{O}-\mathrm{C}$ 伸缩与反伸缩振动)吸收峰逐渐减弱, 在 $220{ }^{\circ} \mathrm{C}$ 完全消 失. $1200 \sim 1100 \mathrm{~cm}^{-1}$ 为 $\mathrm{CF}_{2}$ 的伸缩振动吸收峰, 1172 $\mathrm{cm}^{-1}$ 为 $\mathrm{CF}_{3}$ 的吸收峰. 通过以上 FT-IR 分析, 初步证明 了 M1 单体发生了开环固化反应, 而其固化开环聚合机 理已有详细的报道 ${ }^{[19,20]}$, 所以其反应方程如 Eq. 1 所示. 随着固化温度升高, 在催化剂或热作用下 $\mathrm{C}-\mathrm{O}$ 键断裂. 同时苯环 6 位上的 $\mathrm{H}$ 被取代形成 1,2,3-苯环邻位取代, 并伴随大量酚羟基产生，最终形成交联网状结构。

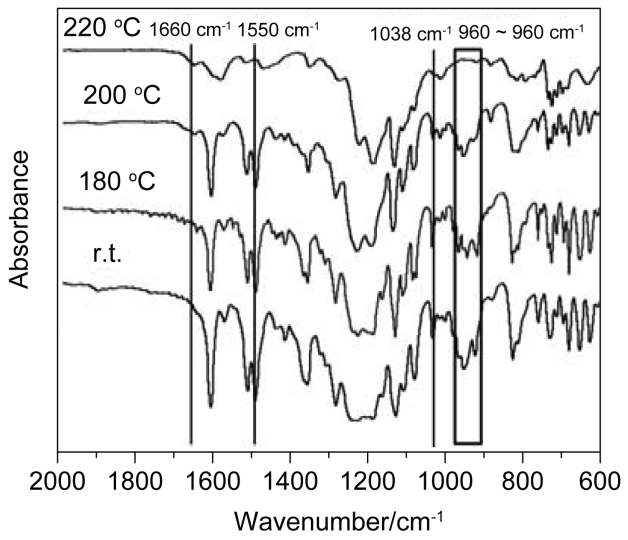

图 3 不同固化温度 M1 的 FT-IR 图谱

Figure 3 FT-IR spectra of M1 curing at different temperatures

\section{3 聚苯并噁嗪性能的研究}

\subsection{1 热稳定性}

通过热重分析(TGA)对含 M1 聚合物的热稳定性进 行了研究, 在氮气氛中其热重损失曲线和分解速率曲线 如图 4 所示. M1 聚合物的 5\%和 10\%的热重损失温度分 别为 337 和 $382{ }^{\circ} \mathrm{C}$, 碳残余率为 $45.9 \%$. 与结构类似的 不含氟长烷基的苯并噁嗪相比, 如聚 ARH12-BA 和聚

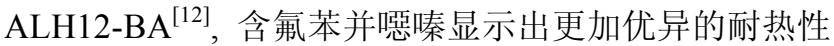
能. 从其分解速率曲线可以看到, 其主要热损失温度在 $350{ }^{\circ} \mathrm{C}$ 至 $600{ }^{\circ} \mathrm{C}$ 之间, 这区间主要为 $\mathrm{C}-\mathrm{H}, \mathrm{C}-\mathrm{C}$ 和 $\mathrm{C}-\mathrm{N}$ 在高温下分解. 而 $\mathrm{C}-\mathrm{F}$ 键能要高于 $\mathrm{C}-\mathrm{H}$ 键, 需 要在更高温度下才能分解. 同时, 由于在 M1 单体结<smiles>[R1]c1ccc(N2COc3ccc(Cc4ccc5c(c4)CN(c4ccc(Br)cc4)CO5)cc3C2)cc1</smiles>

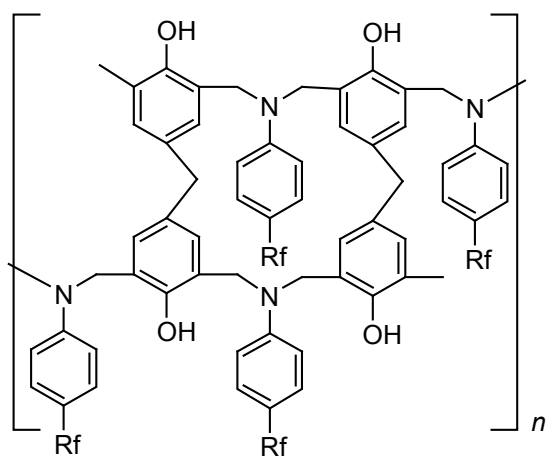

$\mathrm{Rf}=\left(\mathrm{CF}_{2}\right)_{5} \mathrm{CF}_{3}$

构中引入了多个苯环结构, 使得 M1 聚合物的耐热温度 和碳残余率都得到很大提高, 所以侧链含氟聚苯并噁嗪 表现出更好的热稳定性.

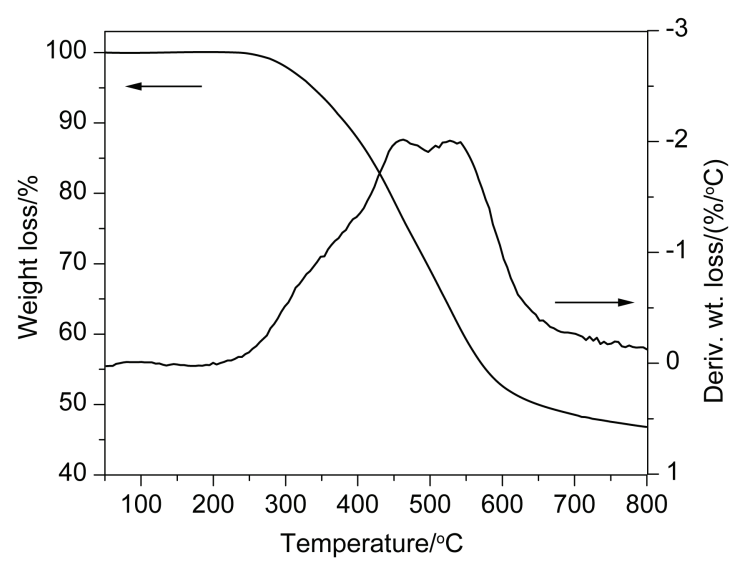

图 4 M1 聚合物的热重损失曲线

Figure 4 TGA analysis of poly(M1)

\subsection{3 介电性能}

由于集成电路元件密度的增加, 高负荷运行给电子 元件信号传导带来一系列问题，如信号延迟和信号干扰 等, 这要求基体材料要具有低介电常数. 因为降低材料 的介电常数可以提高信号传导速率和降低信号传播损 失 ${ }^{[21]}$. 而聚合物的化学结构决定其可极化率, 可极化率 又很大程度影响介电性能 ${ }^{[22]}$. 氟原子具有较低的可极 化率，其电负性为 4.0. 通过使用全氟已基苯胺引入氟 原子, 从而降低苯并噁嗪的极化率并降低介电常数. 常 温下在 $1 \mathrm{MHz}$ 频率, $\mathbf{M} 1$ 聚合物介电常数为 3.78 , 介电损 耗为 0.047 . 相比于含有不饱和、共轭或者极性酚羟基结 构的聚合物, 含有饱和的碳氢键、碳氟键的聚合物具有 更低的极化率 ${ }^{[12]}$. 由于吸电子诱导作用，引入的 
$\left(\mathrm{CF}_{2}\right)_{5} \mathrm{CF}_{3}$ 基团明显地降低了 $\mathrm{M1}$ 聚合物的可极化率. 同 时, 体积庞大的全氟已基, 由于位阻作用降低了分子堆 积, 增加了自由体积. 这些因素都有利于介电常数的降 低.

\subsection{2 吸水率}

水具有很高的介电常数, 在 $25{ }^{\circ} \mathrm{C}$ 时为 78.5. 所以, 低介电材料具有较低的吸水率 $(<1 \%)$ 至为重要. 表 1 中 分别列出了 M1 聚合物常温下在湿度为 $100 \%$ 的空气中 和水中的吸水率. 结果显示, 在不同环境下聚合物的吸 水率几乎都低于 $1 \%$. 从数据可以看出, 高度氟化的聚 苯并噁溙具有很低的吸水率.

表 1 常温下 M1 聚合物的吸水率

Table 1 Water absorption of poly(M1) at room temperature

\begin{tabular}{ccccc}
\hline \multicolumn{2}{c}{ In air } & & \multicolumn{2}{c}{ In water } \\
\cline { 4 - 5 } \cline { 4 - 5 } 1 day & 1 week & & 1 day & 1 week \\
\hline$<1 \%$ & $<1 \%$ & & $<1 \%$ & $2 \%$ \\
\hline
\end{tabular}

\subsection{2 聚合物表面性能}

含氟聚合物能被广泛的应用, 其中一个优异的性能 就是良好的表面性能. 决定聚合物表面性能的因素众 多, 比如聚合物结构中的氢键 ${ }^{[23]}$ 、氟原子的电负性或者 表面形态 ${ }^{[24]}$ 等. 图 5 显示了 $\mathbf{M 1}$ 聚合物样品的接触角. 常温下, 以水和甲酰胺为润湿液, 测得的接触角分别为 $122^{\circ}$ 和 $110^{\circ}$, 通过杨氏方程 ${ }^{[25]}$ 计算可以得到含氟聚苯并 噁嗪的表面自由能为 $6.98 \mathrm{mN} / \mathrm{m}$. 由此表明引入氟原子 可以降低聚合物的表面自由能, 从而提高其耐水润湿 性, 从另一方面降低了吸水率, 降低了介电常数.

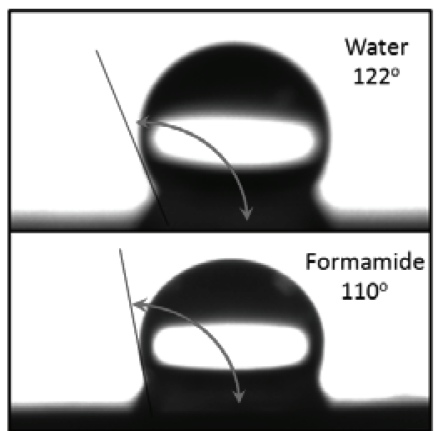

图 5 润湿液水和甲酰胺在 M1 聚合物上的接触角

Figure 5 Measured receding contact angle of poly(M1) used water and formamide as wetting liquid

\section{2 结论}

成功地合成了侧链含长氟碳链苯并噁嗪 $\mathbf{M 1}$, 并通 过 ${ }^{1} \mathrm{H}$ NMR, ${ }^{19} \mathrm{~F}$ NMR, ${ }^{13} \mathrm{C}$ NMR 和 FT-IR 对其结构进行 了表征. 在固化反应中, 加入固化剂咪唑可以很大程度 降低苯并噁嗪的固化温度, 其固化起始温度从 $257{ }^{\circ} \mathrm{C}$ 降低至 $183{ }^{\circ} \mathrm{C}$. 通过引入含氟烷基侧链, 使聚苯并噁嗪
的热稳定性进一步提高，其 5\%和 10\%的热重损失温度 分别为 337 和 $382{ }^{\circ} \mathrm{C}$, 碳残余率为 $45.9 \%$. 同时 M1 聚 合物具有较低的吸水率 $(<1 \%)$ 和表面自由能 $(6.98$ $\mathrm{mN} / \mathrm{m})$, 这都有利于聚合物的介电常数的降低, 测得的 介电常数为 3.78. 这些良好的性能使得 M1 聚合物成为 一种优异的电子封装材料.

\section{3 实验部分}

\section{1 实验原料}

水杨醛(AR，98\%)、多聚甲醛 $(96 \%)$ 和嗍氢化钠 (96\%), 国药试剂公司; 电解铜粉 $(99.9 \%) 、 4$-碘苯胺 (99\%)咪唑(AR，99\%)和 4,4'-二氨基苯甲烷(97\%)，阿拉 丁试剂公司; 全氟碘代烷(工业级, 未处理), 阜新恒通 氟化学试剂公司; $37 \%$ 甲醛溶液(分析纯), 上海凌峰化学 试剂公司; 4-全氟己烷苯胺 ${ }^{[22]}$ 和 5,5'-亚甲基水杨醛 ${ }^{[23]}$ 均 为实验室自制.

\section{2 测试与表征仪器}

核磁氢谱氟谱采用 Mercury 300 型核磁共振仪, 碳 谱采用 Bruker AM-300 核磁共振仪; 示差扫描量热法 (DSC)采用 TA Q200 示差扫描量热仪, 称取 $3 \mathrm{mg}$ 左右样 品于铝盘中, 氮气流量为 $50 \mathrm{~mL} / \mathrm{min}$, 以 $10 \mathrm{~K} / \mathrm{min}$ 的升 温速率从室温升至 $300{ }^{\circ} \mathrm{C}$; 热重损失(TGA)采用耐驰 TG 209 F1 型热重损失分析仪, 称取 $10 \mathrm{mg}$ 左右样品于 氧化铝坩埚中, 氮气流量为 $30 \mathrm{~mL} / \mathrm{min}$, 以 $10 \mathrm{~K} / \mathrm{min}$ 的 升温速率从室温升至 $800{ }^{\circ} \mathrm{C}$; 红外光谱采用 NICOLET 380 FTIR 光谱仪, $\mathrm{KBr}$ 压片, 扫描范围 $500 \sim 4000 \mathrm{~cm}^{-1}$; 介电性能采用 BDS40 (Novocontrol $\mathrm{GmbH}$ ) 高分辨分析 仪, 使用频率为 $1 \mathrm{MHz}$, 常温测试, 将测试样品打磨处 理为直径 $2.2 \mathrm{~cm}$, 厚度为 $2 \sim 3 \mathrm{~mm}$ 的固体圆片; 接触角 采用 POWEREACH JC2000C 型接触角仪, 常温测试, 以水和甲酰胺为润湿液, 分别滴加在未处理的固化样品 表面, 取其截面图片, 计算接触角.

\section{3 单体的合成}

3.3.14,4'-亚甲基双\{2-[4-(全氟已基)苯基亚氨基甲 基]苯酚 $\}$ (3)的合成

向 $100 \mathrm{~mL}$ 两口瓶中加入 $1.56 \mathrm{~g}(6 \mathrm{mmol}) 5,5^{\prime}$-亚甲 基水杨醛和 $50 \mathrm{~mL}$ 的无水乙醇, 加热到回流. 缓慢滴加 $5 \mathrm{~g}(12 \mathrm{mmol}) 4$-全氟已烷苯胺, 反应 $0.5 \mathrm{~h}$, 反应体系有 大量黄色固体析出. 反应 $2 \mathrm{~h}$ 后, 停止反应, 自然冷却, 过滤, 丙酮重结晶两次, 得到黄色固体粉末 $4.93 \mathrm{~g} \mathrm{(4.67}$ $\mathrm{mmol})$, 反应产率为 $77.8 \% .{ }^{1} \mathrm{H} \mathrm{NMR}\left(300 \mathrm{MHz}, \mathrm{CDCl}_{3}\right)$ $\delta: 12.74(\mathrm{~s}, 2 \mathrm{H}, \mathrm{OH}), 8.59(\mathrm{~s}, 2 \mathrm{H}, \mathrm{CH}=\mathrm{N}), 7.64(\mathrm{~d}, J=6$ Hz, 4H, Ar-H), 7.35 (d, J=9 Hz, 4H, Ar-H), 7.29 (s, 1H, Ar-H), 7.20 (s, 2H, Ar-H), 7.02 (d, $J=9$ Hz, 3H, Ar-H), 
$3.96\left(\mathrm{~s}, 2 \mathrm{H}, \mathrm{CH}_{2}\right) ;{ }^{19} \mathrm{~F}$ NMR $\left(282 \mathrm{MHz}, \mathrm{CDCl}_{3}\right) \delta$ : $-81.19(\mathrm{t}, J=17.77 \mathrm{~Hz}, 3 \mathrm{~F}),-110.91(\mathrm{t}, J=26.51 \mathrm{~Hz}$, $2 \mathrm{~F}),-121.96(\mathrm{~s}, 2 \mathrm{~F}),-122.36(\mathrm{~s}, 2 \mathrm{~F}),-123.34(\mathrm{~s}, 2 \mathrm{~F})$, -126.68 (s, 2F). Anal. calcd for $\mathrm{C}_{39} \mathrm{H}_{20} \mathrm{~F}_{26} \mathrm{~N}_{2} \mathrm{O}_{2}$ : C 44.93, H 1.93, N 2.68; found C 44.80, H 1.99, N 2.67.

$3.3 .24,4$-亚甲基双 \{2-[4-(全氟已基)苯基氨基甲基] 苯酚\}(4)的合成

将 $17 \mathrm{~g}$ (16.31 mmol) 3 加入到装有 $100 \mathrm{~mL}$ 无水乙 醇的单口烧瓶中, 常温下搅拌 $5 \mathrm{~min}$. 然后嗍氢化钠每 隔 $0.5 \mathrm{~h}$ 加一次, 分三次加入 $(0.64 \mathrm{~g} \times 3)$. 反应 $2 \mathrm{~h}$ 后, 待 溶液清亮. 将反应液倒入水中, 析出白色浑浊物. 乙酸 乙酯萃取, 无水硫酸钠干燥, 过滤, 旋干溶剂. 得到白

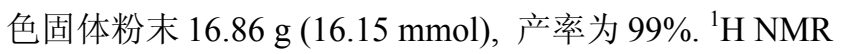
(300 MHz, DMSO- $\left.d_{6}\right) \delta: 9.40(\mathrm{~s}, 2 \mathrm{H}, \mathrm{OH}), 7.25$ (d, $J=9$ $\mathrm{Hz}, 4 \mathrm{H}, \mathrm{Ar}-\mathrm{H}), 6.98$ (s, 2H, Ar-H), 6.82 (d, J=15 Hz, 2H, Ar-H), $6.74 \sim 6.67(\mathrm{~m}, 6 \mathrm{H}, \mathrm{Ar}-\mathrm{H}), 5.76(\mathrm{t}, J=12 \mathrm{~Hz}, 2 \mathrm{H}$, $\mathrm{NH}), 4.15\left(\mathrm{~d}, J=3 \mathrm{~Hz}, 2 \mathrm{H}, \mathrm{Ar}-\mathrm{CH}_{2}-\mathrm{N}\right), 3.61\left(\mathrm{~s}, 2 \mathrm{H}, \mathrm{CH}_{2}\right)$; ${ }^{13} \mathrm{C}$ NMR (100 MHz, DMSO- $\left.d_{6}\right) \quad \delta: 155.45,147.33$, $129.87,129.38,128.66,127.84,126.42,119.23,115.32$, $112.73,42.16,40.14 ;{ }^{19} \mathrm{~F}$ NMR (282 MHz, DMSO- $\left.d_{6}\right) \delta$ : $-81.06(\mathrm{~d}, J=28.20 \mathrm{~Hz}, 3 \mathrm{~F}),-108.32(\mathrm{~s}, 2 \mathrm{~F}),-121.98$ $(\mathrm{s}, 2 \mathrm{~F}),-122.25(\mathrm{~s}, 2 \mathrm{~F}),-123.32(\mathrm{~s}, 2 \mathrm{~F}),-126.57(\mathrm{~s}$, 2F). Anal. calcd for $\mathrm{C}_{39} \mathrm{H}_{22} \mathrm{~F}_{26} \mathrm{~N}_{2} \mathrm{O}_{2}: \mathrm{C} 44.84, \mathrm{H} 2.12, \mathrm{~N}$ 2.68; found C 44.80, H 2.41, N 2.57.

\subsection{3 含氟苯并噁嗪 M1 的合成}

将 $15 \mathrm{~g}$ (14 mmol) 4 和 $100 \mathrm{~mL}$ 的氯仿加入到 250 $\mathrm{mL}$ 的两口瓶中, 缓慢滴加 $2.4 \mathrm{~g}$ 的 $37 \%$ 甲醛溶液 $(30$ $\mathrm{mmol}$ ). 加热到回流, 反应 $2 \mathrm{~h}$ 后, 冷却至常温. 将反应 液用蒸馏水洗三遍, 无水硫酸钠干燥, 过滤, 旋干溶剂. 得到白色粉末固体 $15.06 \mathrm{~g}$ (13.80 mmol), 产率为 $98 \%$. m.p. $133{ }^{\circ} \mathrm{C}$; ${ }^{1} \mathrm{H}$ NMR (300 MHz, $\left.\mathrm{CDCl}_{3}\right) \delta: 7.45$ (d, $J=9$ $\mathrm{Hz}, 4 \mathrm{H}, \mathrm{Ar}-\mathrm{H}$ ), 7.13 (d, $J=6 \mathrm{~Hz}, 4 \mathrm{H}, \mathrm{Ar}-\mathrm{H}), 6.95$ (d, $J=6$ $\mathrm{Hz}, 2 \mathrm{H}, \mathrm{Ar}-\mathrm{H}), 6.79$ (d, J=6 Hz, 3H, Ar-H), 6.75 (s, 1H, $\mathrm{Ar}-\mathrm{H}), 5.36\left(\mathrm{~s}, 4 \mathrm{H}, \mathrm{OCH}_{2} \mathrm{~N}\right), 4.64$ (s, 4H, $\left.\mathrm{ArCH}_{2} \mathrm{~N}\right), 3.79$ $\left(\mathrm{s}, 2 \mathrm{H}, \mathrm{CH}_{2}\right) ;{ }^{13} \mathrm{C} \mathrm{NMR}\left(100 \mathrm{MHz}, \mathrm{CDCl}_{3}\right) \delta: 152.65$, $151.05,134.07,128.61,128.20,126.84,120.44,117.19$, 116.54, 77.87, 49.97, 40.35; ${ }^{19} \mathrm{~F}$ NMR (282 $\left.\mathrm{MHz}, \mathrm{CDCl}_{3}\right)$ $\delta$ : $-81.25(\mathrm{t}, J=21.43 \mathrm{~Hz}, 3 \mathrm{~F}),-110.35(\mathrm{t}, J=31.58$
$\mathrm{Hz}, 2 \mathrm{~F}),-121.97$ (s, 2F), - 122.33 (s, 2F), - 123.29 (s, 2F), -126.61 (s, 2F); IR (KBr) v: 2904, 1610, 1510, 1229, 1200, 1172, 1100, 1038, 942; HRMS (ESI) calcd for $\mathrm{C}_{41} \mathrm{H}_{24} \mathrm{~N}_{2} \mathrm{O}_{2} \mathrm{~F}_{26}$ 1070.6, found 1070.7. Anal. calcd for $\mathrm{C}_{41} \mathrm{H}_{24} \mathrm{~F}_{26} \mathrm{~N}_{2} \mathrm{O}_{2}$ : C 46.01, H 2.26, N 2.62; found C 45.78, $\mathrm{H}$ 2.38, N 2.61.

\section{$3.4 \mathrm{M} 1$ 聚合物的制备}

称取一定量的 M1 单体与质量分数为 $5 \%$ 的固化剂 咪唑, 置于玛瑙研钵中进行研磨, 充分混合均匀. 然后 将粉末倒入模具中, $100{ }^{\circ} \mathrm{C}$ 真空干燥 $2 \mathrm{~h}$ 以除去低沸点 溶剂. 在氩气保护下，按如下程序升温: $170{ }^{\circ} \mathrm{C} / 1 \mathrm{~h}, 180$ ${ }^{\circ} \mathrm{C} / 1 \mathrm{~h}, 190{ }^{\circ} \mathrm{C} / 1 \mathrm{~h}, 200{ }^{\circ} \mathrm{C} / 1 \mathrm{~h}, 210{ }^{\circ} \mathrm{C} / 1 \mathrm{~h}, 220{ }^{\circ} \mathrm{C} / 1 \mathrm{~h}$, 然后自然冷却至常温, 得到 M1 聚合物.

\section{References}

[1] Ishida, H.; Low, H. Y. Macromolecules 1997, 30, 1099.

[2] Ning, X.; Ishida, H. J. Polym. Sci.: Part B: Polym. Phys. 1994, 32, 921.

[3] Ishida, H.; Allen, D. J. Polym. Sci.: Part B: Polym. Phys. 1996, 34, 1019.

[4] Takeichi, T.; Zeidam, R.; Agag, T. Polymer 2002, 43, 45.

[5] Lee, Y. J.; Huang, J. M.; Kuo, S. W. Polymer 2005, 46, 2320.

[6] Ishida, H.; Low, H. Y. J. Appl. Polym. Sci. 1998, 69, 2559.

[7] Mantana, K.; Nantaya, Y. Polym. Polym. Compos. 2001, 9, 367.

[8] Su, Y. C.; Chang, F. C. Polymer 2003, 44, 7989.

[9] Lin, C. H. J. Polym. Sci., Part A: Polym. Chem. 2008, 46, 4970.

[10] Lui, J. P.; Ishida, H. Polym. Polym. Compos. 2002, 10, 191.

[11] Pedro, V. H.; Ishida, H. J. Fluorine Chem. 2009, 13, 573.

[12] Pedro, V. H. Macromolecules 2008, 41, 9704.

[13] Shi, Z. X.; Pan, Y.; Wang, Y. Z; Yu, D. S. J. Beijing Univ. Chem. Technol. 2000, 27, 13 (in Chinese).

(史子兴, 潘颖, 王一中, 余鼎声, 北京化工大学学报, 2000, 27, 13.)

[14] Chen, X. P. RCS Adv. 2012, 2, 6504.

[15] Lin, C.H.; Chang, S. L. Polymer 2008, 49, 1220.

[16] Kureshy, R. I. Tetrahedron: Asymmetry 2001, 12, 433.

[17] Mohan, N. W.; Wolter, F. J. J. Org. Chem. 2010, 75, 6814.

[18] Brunovska, Z.; Liu, J. Macromol. Chem. Phys. 1999, 200, 1745.

[19] Kim, H. D.; Ishida H. Macromolecules 2003, 36, 8320.

[20] Liu. C.; Shen D. M. Macromolecules 2011, 44, 4616.

[21] Maier, G. Prog. Polym. Sci. 2001, 26, 3.

[22] Hougham, G.; Tesoro, G.; Viehbeck, A.; Chapple-Sokol, J. D. Macromolecules 1994, 27, 5964.

[23] Sun, T.; Wang, G.; Feng, L. Angew. Chem. 2004, 116, 361.

[24] Sehonhorn, H. Macromolecules 1968, 1, 145.

[25] Katano, Y.; Tomono, H. Macromolecules 1994, 27, 2342.

(Cheng, F.) 